\section{LL-06 PROVIDER PERCEPTIONS ON THE MANAGEMENT OF LUPUS DURING PREGNANCY: BARRIERS TO IMPROVED CARE}

Megan EB Clowse*, Jessica Revels, Amanda Eudy, Gillian Schmidler-Sanders, Laura Neil, Jennifer Gierisch. Duke University School of Medicine, Durham, North Carolina

\subsection{6/lupus-2018-Ism.116}

Background More than half of pregnancies in women with systemic lupus erythematosus (lupus) result in adverse outcomes for both mother and fetus. As demonstrated in the PROMISSE study, pregnancies managed by experts can have outcomes similar to the general population. However, many women with lupus do not receive this level of care and suffer complications at higher rates. We sought to identify aspects of current community rheumatologic care that could be improved to decrease the frequency of poor outcomes.

Methods Focus groups with clinical rheumatologists were held at state rheumatology conferences in North Carolina, South Carolina, Virginia, and Northern California. The interview guide was based on the PRECEDE/PROCEED framework, seeking factors that predisposed, reinforced, and enabled physician practice patterns. Focus groups were recorded, transcribed, and coded to identify the elements of this framework. A group of women with lupus on their reproductive journey contributed to our understanding of the dilemmas and care provided.

Results Medically ill-timed pregnancies and medication nonadherence during pregnancy were identified by the rheumatologists as the 2 key dilemmas in care. We identified several communication gaps as a key modifiable barrier to optimal management: 1) the approach to physician/patient communication was often ill-suited to the sensitive discussion about pregnancy planning; 2) the communication of treatment plans was often hampered by gaps in knowledge and confidence in data, encouraging non-adherence among nervous patients; and 3) local rheumatologists and OB/GYN frequently did not communicate, leading to varying treatment plans and confusion for patients.

The key predisposing factors that impaired care included gaps in knowledge and skills in discussing pregnancy planning and management, as well as clinic time for repeated discussions. Reinforcing factors that influenced care included local treatment factors and concerns over legal consequences. Factors that could enable improved care included access to an expert or guidelines, clinical reminders, and patient handouts. Conclusions Our goal is to ensure that all women with lupus across the country receive expert-level care for pregnancy planning and management by elevating the care received from community rheumatologists. This study demonstrated the importance of communication skills, knowledge, and cohesive plans between community physicians in reaching this goal. We are currently developing HOP-STEP (Healthy Outcomes for Pregnancies in SLE Through Educating Providers and Patients), a multifaceted implementation project to enable women with lupus and their rheumatologists to have honest and accurate conversations about pregnancy planning and management.

Acknowledgements The Duke Autoimmunity in Pregnancy Patient Advisors and Collaborators contributed to this abstract.

\section{LL-07 SELF-MANAGEMENT ABILITY IS LOW IN ONE THIRD OF A SAMPLE OF PATIENTS WITH SYSTEMIC LUPUS ERYTHEMATOSUS}

${ }^{1}$ Paul R Fortin*, ${ }^{2}$ Carolyn Neville, ${ }^{3}$ Anne-Sophie Julien, ${ }^{4}$ Murray Rochon, ${ }^{5}$ Elham Rahme, ${ }^{6}$ Vinita Haroun, ${ }^{2}$ Deborah DaCosta. 'Division of Rheumatology, Department of Medicine, CHU de Québec - Université Laval, Quebec City, Canada; ${ }^{2}$ Centre for Outcomes Research and Evaluation, Research Institute of the McGill University Health Centre, Montreal, Canada; ${ }^{3}$ Plateforme de recherche clinique, Centre de recherche du CHU de Québec - Université Laval, Quebec City, Canada; ${ }^{4}$ Jack Digital Productions, Inc., Toronto, Canada; ${ }^{5}$ Division of Clinical Epidemiology, McGill University Health Centre, Montreal, Canada; ${ }^{6}$ Research Committee, Lupus Canada, Newmarket, Canada

\subsection{6/lupus-2018-Ism.117}

Background MyLupusGuide is a web-based lupus interactive navigator that aims to improve patient's confidence in their decision making about their lupus care.

Methods MyLupusGuide was modeled on the Oncology Interactive Navigator $^{\mathrm{TM}}$ with input from persons living with lupus and members of their care teams. We are conducting a randomized dissemination trial to measure the impact of MyLupusGuide on patient activation and other key psychosocial indicators. A letter of invitation was mailed out by local investigators of the Canadian Network for Improved Outcomes in SLE (CaNIOS) with information on how to enter the website. An online consent was obtained upon registration. Once the baseline questionnaires were completed, one group obtained immediate unlimited access to MyLupusGuide while the other group was informed that they would access it three months later. Besides collecting sociodemographic and selfreported disease and health status measures, we collected information on the Patient Activation Measure ${ }^{\mathrm{TM}}$ (PAM), a valid instrument to measure self-management ability. PAM levels 1 and 2 out of a 4 level scale indicate low activation that translate into being overwhelmed with poor goal orientation,

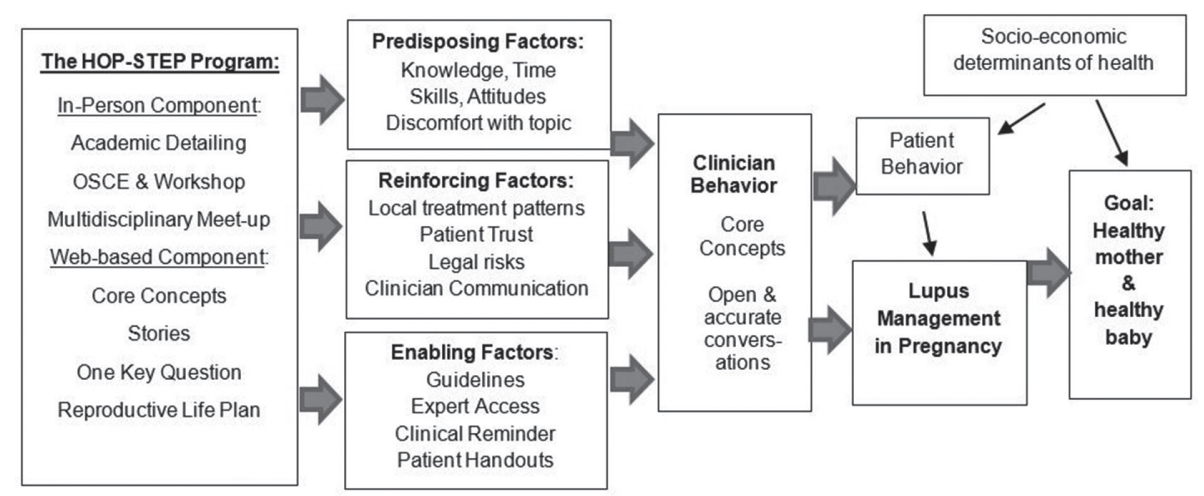

Abstract LL-06 Figure 1 The PRECEDE/PROCEED model underlying the HOP-STEP program 
negative disposition, weaker support system, poor problemsolving skills, unclear on their healthcare role, significant knowledge gaps, and poor self-management skills. We report here on the baseline data. Descriptive statistics were performed using Wilcoxon Mann Whitney Test, Exact Pearson Chi Square Test, or a Pearson Chi Square Test, as appropriate. Results We sent letters to 1916 patients affiliated with 10 CaNIOS centers in January 2018. A total of 571 patients responded and $541(28.2 \%)$ completed all the baseline questionnaires. The average age was 50 (range 19-85), 93\% were female with an average duration of lupus of 17 years. Three quarters of respondents were Caucasian, 55\% had a college or university degree and 64\% were working while 26\% reported being on disability. We report in table 1 the self-reported disease characteristic at baseline using the Systemic Lupus Activity Questionnaire (SLAQ), the Lupus Damage Index Questionnaire (LDIQ) and the physical and mental component summary scores of the SF-36 $6^{\mathrm{TM}}$, the number of computer hour usage, the frequency of searching for medical information and the PAM. We can observe that one third (35\%) of our patients have a low PAM score at baseline.

Conclusions We observed low patient self-management ability in one third of a large sample of lupus patients participating into a trial of MyLupusGuide suggesting that this vulnerable population needs additional resources to improve their confidence in self-managing their lupus.

\begin{tabular}{|c|c|c|c|c|c|}
\hline Characteristic & $\begin{array}{l}\text { Group A } \\
(n=265)\end{array}$ & $95 \% \mathrm{Cl}$ & $\begin{array}{l}\text { Group B } \\
(n=276)\end{array}$ & $95 \% \mathrm{Cl}$ & $\mathrm{p}$-value \\
\hline \multicolumn{6}{|l|}{ SLAQ } \\
\hline Mean (sd) & $13.0(7.6)$ & $\begin{array}{l}(12.1 ; \\
14.0)\end{array}$ & $14.9(8.3)$ & $\begin{array}{l}(14.0 ; \\
15.9)\end{array}$ & $0,0125^{*}$ \\
\hline \multicolumn{6}{|l|}{ LDIQ } \\
\hline $\begin{array}{l}\text { Mean (sd) } \\
\text { SF36 }\end{array}$ & $3.3(2.9)$ & $(3.0 ; 3.7)$ & $3.7(3.2)$ & $(3.4 ; 4.1)$ & 0,0952 \\
\hline PC mean (sd) & $40.2(11.5)$ & $\begin{array}{l}(38.8 ; \\
41.6)\end{array}$ & $37.7(12.2)$ & $\begin{array}{l}(36.3 ; \\
39.2)\end{array}$ & $0,0266^{*}$ \\
\hline MC mean (sd) & $44.4(11.2)$ & $\begin{array}{l}(43.0 ; \\
45.7)\end{array}$ & $43.6(11.4)$ & $\begin{array}{l}(42.3 ; \\
45.0)\end{array}$ & 0,4103 \\
\hline \multicolumn{6}{|c|}{ Computer usage (hours/week) } \\
\hline Mean (sd) & $15.5(15.8)$ & $\begin{array}{l}(13.6 ; \\
17.5)\end{array}$ & $13.4(11.3)$ & $\begin{array}{l}(12.1 ; \\
14.8)\end{array}$ & 0,1184 \\
\hline \multicolumn{6}{|c|}{$\begin{array}{l}\text { Frequency of searching for medical } \\
\text { information (\%) }\end{array}$} \\
\hline Never & $6(2)$ & & $12(4)$ & & 0,2078 \\
\hline Sometimes & $154(59)$ & & $143(53)$ & & \\
\hline Frequently & 74 (28) & & 91 (34) & & \\
\hline Very frequently & $28(11)$ & & $23(9)$ & & \\
\hline PAM & & & & & 0,7788 \\
\hline Mean (sd) & $61.1(13.6)$ & $\begin{array}{l}(59.4 ; \\
62.7)\end{array}$ & $61.1(14.0)$ & $\begin{array}{l}(59.4 ; \\
62.7)\end{array}$ & \\
\hline Level 1 n (\%) & $39(14.7)$ & & $49(17.8)$ & & \\
\hline Level 2 n (\%) & $57(21.5)$ & & $50(18.2)$ & & \\
\hline Level 3 n (\%) & $111(41.9)$ & & $114(41.5)$ & & \\
\hline Level $4 \mathrm{n}(\%)$ & $58(21.9)$ & & $62(22.5)$ & & \\
\hline
\end{tabular}

Acknowledgements We thank the Canadian Institutes for Health Research and their Knowledge-to-Action grant program for supporting this trial. We also thank the individual CaNIOS centers and their local investigators, research assistants and patients that have made this study possible.

Trial registration The trial registration numbers are NCT02950714 and KAL-356634.

\section{LL-08 WALK SLE - END OF STUDY RESULTS OF A PILOT STUDY EXPLORING WALK WITH EASE, A SELF-DIRECTED WALKING PROGRAM, IN LUPUS PATIENTS}

${ }^{1}$ Brittaney-Belle E Gordon, ${ }^{2}$ Katherine Kaufman, ${ }^{1}$ Sean T Hicks, ${ }^{1}$ Rebecca J Cleveland, ${ }^{1} J u l i e$ A Norfleet, 'Leigh F Callahan, 'Saira Z Sheikh*. 'University of North Carolina at Chapel Hill School of Medicine, Chapel Hill, NC, USA; ${ }^{2}$ Duke University School of Medicine, Durham, NC, USA

\subsection{6/lupus-2018-Ism.118}

Background Fatigue and arthritis are common sources of impairment in SLE patients that persist despite lifestyle and pharmacologic interventions. Walk with Ease (WWE) is the Arthritis Foundation's 6 week evidence-based walking program developed for adults with arthritis that has been shown to improve physical function, pain, stiffness and fatigue in individuals with arthritis. WWE is offered in two formats - group (instructor led) or self-directed. The goal of this study was to examine the feasibility of self-directed WWE in SLE patients.

Methods We enrolled 75 SLE patients to take part in the WWE program, all of whom completed an initial evaluation. The post- 6 week evaluation with no usual care comparison group was conducted in 46 patients. Self-reported outcomes including symptoms (pain, stiffness and fatigue visual analog scales [VAS]) and Functional Assessment of Chronic Illness Therapy-Fatigue (FACIT-fatigue) scale were evaluated. Means and effect sizes (ES) with 95\% confidence intervals were computed for changes in symptoms between baseline and 6 weeks, adjusted for gender, age, race, and baseline outcome. Participant satisfaction measures with WWE are reported as percentages.

Results Participants were mainly women (96.0\%), aged $\geq 40$ years, self-identified as black (53.3\%) and 13 years from initial SLE diagnosis. Fifteen (20.0\%) participants also had a physician diagnosis of OA, while $21(28.0 \%)$ participants had concurrent fibromyalgia. Four participants were screen failures, 9 were withdrawn by the study team, 13 were lost to follow-up and 3 had not yet reached the end of the 6 week protocol. There were 46 participants who completed the WWE program; no significant differences in baseline measures were observed between those who did and did not complete the study. At the end of 6 weeks, participants reported having reduced FACIT-fatigue scores $(\mathrm{ES}=0.14)$, as well as having reduced scores for VAS fatigue and stiffness $(\mathrm{ES}=0.22$ and 0.11 , respectively [table 1]). Most participants reported walking for at least 30 minutes/day (47.7\%), $\geq 3$ days/week $(86.4 \%)$ and utilized the workbook for motivation (65.3\%). Participants also reported increased physical activity (81.8\%), confidence to continue exercising (97.8\%) and satisfaction from the program $(97.8 \%)$. 